


\section{Religionswissenschaft und Theologie}

\section{aus der Sammlung Göschen}

VERGLEICHENDE RELIGIONSWISSENSCHAFT. Von Prof. D. Dr.

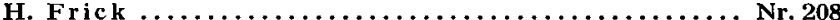
RELIGIONSPHilosopHiE. Von Prof. Dr. otto v. d. Pf orden Nr. 772 PSYCHOLOGIE DER RELIGION, Von Dr. Richard Müller-Freien-

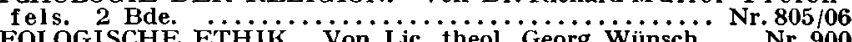
THEOLOGISCHE ETHIK. Von Lic. theol. Georg Wünsh.... Nr. 900 GESCHICHTE DES ALTEN MORGenlandes. Von Prof. Dr. E.

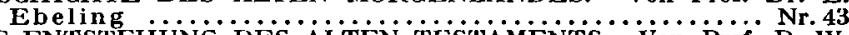
DIE ENTSTEHUNG DES ALTEN TESTAMENTS. Von Prof. D. W.

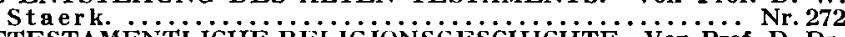
ALTTESTAMENTLiche RELigiónsGeschichte. Von Prof. D. Dr.

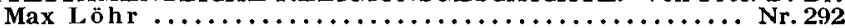
GESCHICHTE ISRAELS bis a. d. griechische Zeit. Von Lic. Dr. J. Ben-

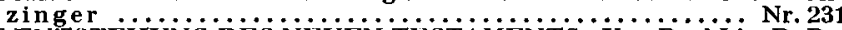

DIE ENTSTEHUNG DES NEUEN TESTAMENTS. Von Paul Lic. D. Dr.

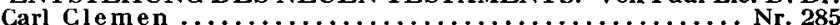

DIE ENTWICKLUNG DER CHRISTLICHEN RELIGION junerhalb des Neuen Testaments. Von Prof. Lic. D. Dr. Carl Clemen.... Nr. 388 NEUTESTAMENTLICHE ZEITGESCHICHTE. Von Prof. Lic. D. Dr. W. S ta erk. 2 Bde................................ $325 / 26$

GESCHICHTE DER URCHR ISTLICHEN LITERATUR. Von Prof. D.

Dr. Martin Dibelius. 2 Bde................ Nr. 934, 935

GESCHICHTE DER ALTCHRISTLICHEN UND LATEINISCHEN LITERATUR vom 2. bis 6. Jahrhundert. Von Prof. Dr. A. Gudemann. Nr. 898

DIE CHRISTLICHEN ITTERATUREN DES OR IENTS. Von Dr, Anton

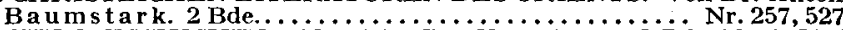
KIRCHENGESCHICH'TE. Von Lic. Dr. Kurt A ner. 3 Bde. Nr. 985/87 DOGMENGESCHICH'E. Von Prof. D. Dr. Friedrich Wiegand. 3 Bde. Nr. $993 / 94,1007$

DAS KIRCHENRECHT. Von Prof. D. Dr. E. Sehling. 2 Bde. Nr. 377,954 PROTESTANTISCHE THEOLOGIE. Uberblick und Einfürung. Von

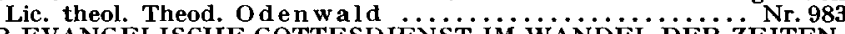

DER EVANGELISCHE GOTTESDIENST IM WANDEL DER ZEITEN.

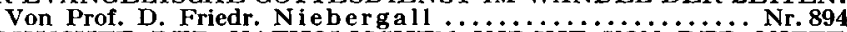

GESCHICHTE DER KATHOLISCHEN K IRCHE VON DER MITTE DES XVIII. JAHRHUNDERTS BIS ZUM VATIKANISCHEN KONZIL. Von Geh. Kons.-Rat Prof. D. Mirbt........ Nr. 700 SYMBOLIK DES RÖMISCHEN KATHOLIZISMUS. Von Pfarrer Dr. Leonhardt Fendt . ....................... 937

DIE KATHOLISCHEN MISSIONEN von der völkerwanderung bis zur Gegenwart. Von Prof. D. Dr. Jos. Schmidlin......... Nr. 913 THEOSOPHIE nebst Anthroposophie und Christengemeinschaft. Von Lic.

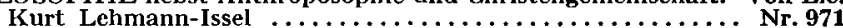

DIE RELIGIONEN DER NATURVÖLKER im Umriß. Von Prof. Dr.

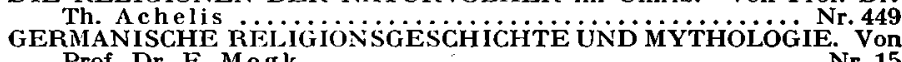
Prof. Dr. E. Mogk. ........................... 15 BUDDHISMUS, (Buddha und seine Lehre). "Von Prof. Dr. Hermann Beckh 2 Bde.

DIE KIRCHE DES MORGENLANDES. (Weltanschauung und Frömmigkeitsleben). Von Prof. $N$. von Arseniew.............. 918 DIE ENTSTEHUNG DES TALMUDS. Von Dr. S. Funk.... Nr. 479 TALMUDPROBEN, Von Dr. S. Funk .................... 583 K'UNGTSE UND DER KONFUZIANISMUS. Von Prof. D. Dr. Richard

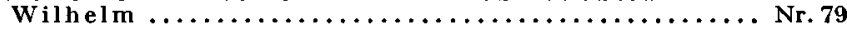

Jeder Band in Leinen geb. $M .8 .80$

Gesamtverzeichnisse und Sonderverzeichnisse liefert Ihnen Ihre Buchhandlung oder der Verlaj unter Bezugnahme auf diese Anzeige gern kostenlos.

Walter de Gruyter \& Co. / Berlin W Io und Leipzig 